Supporting Information

\title{
Relationship between Reaction Kinetics and Chain Dynamics of Vitrimers based on Dioxaborolane Metathesis
}

\author{
Shilong Wu, Huanhuan Yang, Shaoyong Huang, and Quan Chen*
}

Chinese Academy of Sciences, Changchun Institute of Applied Chemistry, State Key

Lab of Polymer Physics and Chemistry, Changchun Institute of Applied Chemistry, Renmin St 5625, Changchun 130022, Jilin, China

*: all correspondence should be sent to qchen@ciac.ac.cn 


\section{Materials}

2,3-Dihydroxypropyl methacrylate was purchased from Aikonchem Co., Ltd. 1,4Phenylenebisboronic acid (Energy chemical, 98\%), 1,2-propanedial (Energy chemical, 99\%) and Phenylboronic acid (Energy chemical, 98\%) were used as received. Hexyl methacrylate (HMA, Aladdin, 98\%) was distilled under reduced pressure to remove inhibitor MEHQ before use. 2-Cyanoprop-2-yl-dithiobenzoate, used as a chain transfer agent (CTA), was purchased from Sinocompound Catalysts Co., Ltd. The polymerization initiator, 2,2'-Azobis(isobutyronitrile) (AIBN, 99\%), was purchased from J\&K, and recrystallized from ethanol before use. Molecular sieves (4 A, Aladdin) was dried in a muffle furnace at $300^{\circ} \mathrm{C}$ for 10 hours before use. Toluene (analytical purity, Beijing Chemical Reagents Co.), tetrahydrofuran (THF, analytical purity, Beijing Chemical Reagents Co.) and dichloromethane (DCM, analytical purity, Beijing Chemical Reagents Co.) were dried with powdered calcium hydride $\left(\mathrm{CaH}_{2}\right)$ and then distilled under reduced pressure or normal pressure before use.

\section{Synthesis of vitrimer samples}

2.1 Synthesis of monomer 2,3-dioxaborolanepropyl methacrylate
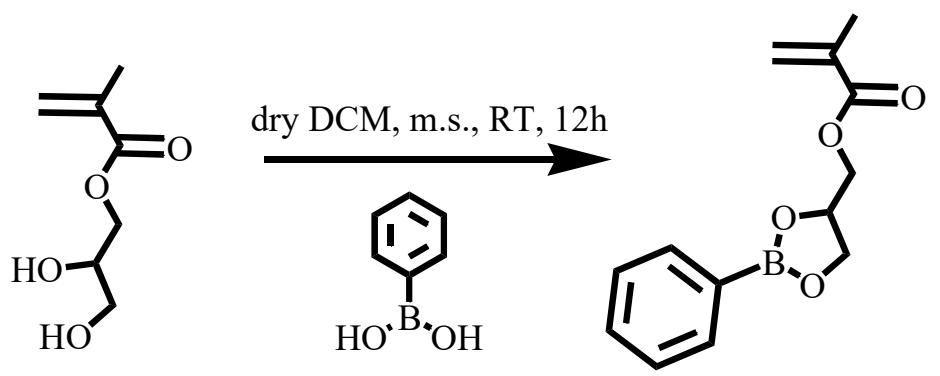

Scheme S1. The synthesis route of precursor

Phenylboronic acid (5g, 41mmol) and 2,3-dihydroxypropyl methacrylate (a, 5.96g, $37 \mathrm{mmol}$ ) were stirred in $100 \mathrm{~mL}$ dry DCM with $15 \mathrm{~g}$ molecular sieves in a flask at room temperature. The process of the reaction was monitored by ${ }^{1} \mathrm{H}$ NMR and ${ }^{13} \mathrm{C}$ NMR spectroscopy. After $12 \mathrm{~h}$ reaction, the raw product was purified through filtering, centrifuging, filtering, and condensation into the final product, a yellow oil sample. The product was characterized by ${ }^{1} \mathrm{H}$ NMR and ${ }^{13} \mathrm{C}$ NMR spectroscopy as shown in Figure S1 and Figure S2. 
${ }^{1} \mathrm{H}$ NMR (400 MHz, $\left.\mathrm{CDCl}_{3}\right): \delta(\mathrm{ppm})$ 7.37-7.83 (m, 5H), $6.11(\mathrm{~s}, 1 \mathrm{H}), 5.56(\mathrm{~s}, 1 \mathrm{H})$, 4.82-4.88 (m, 1H), 4.44-4.49 (t, 1H), 4.34-4.38(dd, 1H), 4.18-4.22(dd, 1H), 1.91(s, 3H). ${ }^{13} \mathrm{C}$ NMR (100 MHz, $\left.\mathrm{CDCl}_{3}\right): \delta$ (ppm) 167.0, 135.5, 134.7, 134.7, 131.4, 127.7, 127.7, 127.7,126.2, 74.7,67.7, 65.6, 18.0. The boron-bound carbon was not detected due to quadrupolar relaxation.

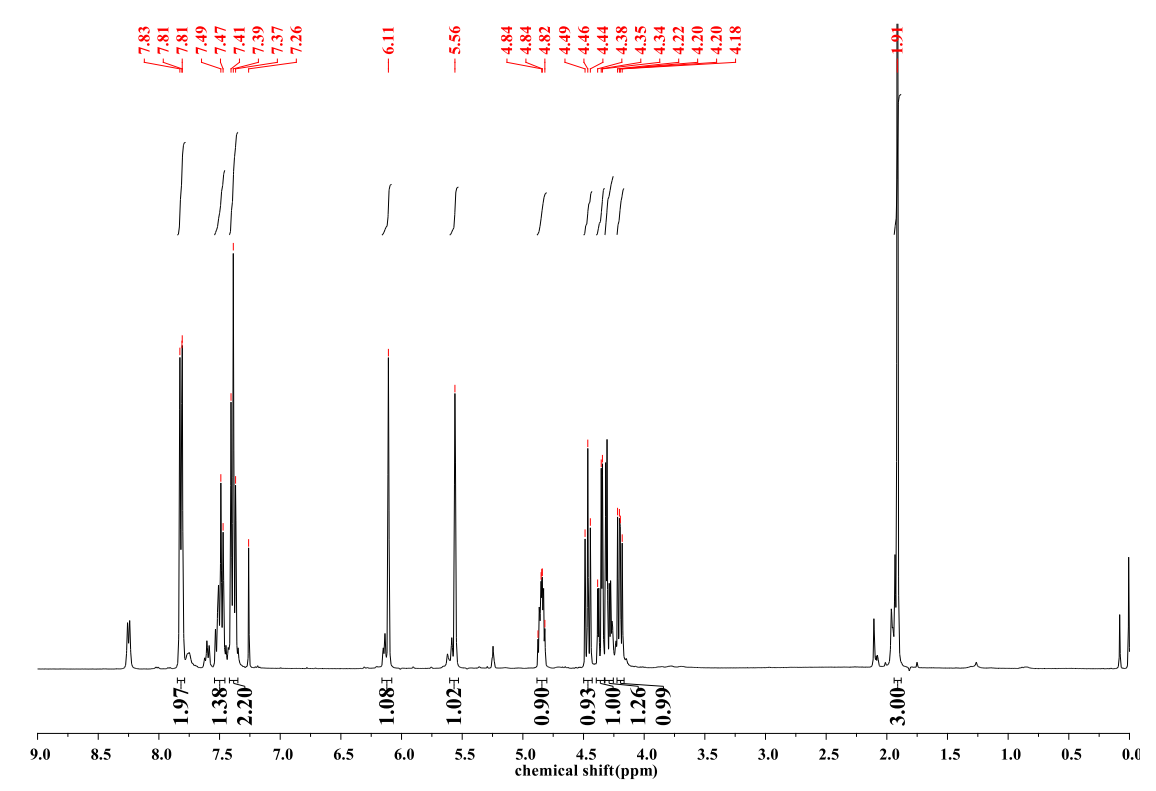

Figure S1. ${ }^{1} \mathrm{H}$ NMR spectra of 2,3-dioxaborolanepropyl methacrylate

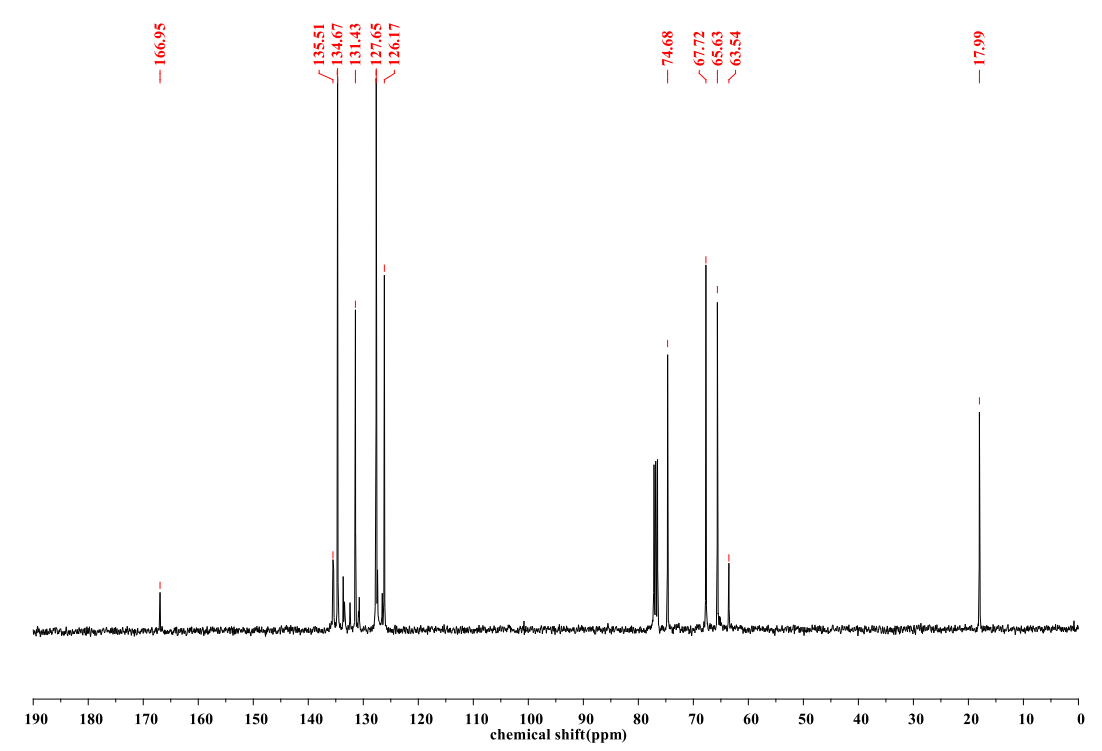

Figure S2. ${ }^{13} \mathrm{C}$ NMR spectra of 2,3-dioxaborolanepropyl methacrylate 
2.2 RAFT copolymerization of precursor, poly (hexyl methacrylate-random-2,3dioxaborolanepropyl methacrylate)

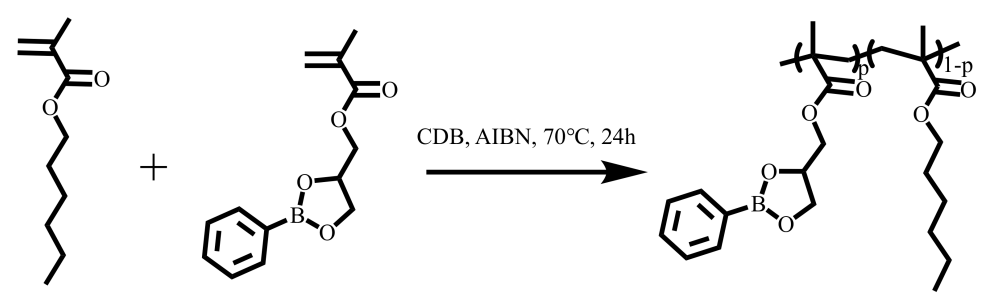

Scheme S2. The synthesis route of precursor

To synthesize the precursor, poly (hexyl methacrylate-random-2,3-dioxaborolane propyl methacrylate), a predetermined amount of purified monomers HMA (3g, $17.6 \mathrm{mmol}), 2,3$-dioxaborolanepropyl methacrylate $(0.277 \mathrm{~g}, 1.1 \mathrm{mmol})$, a chain transfer agent 2-cyanoprop-2-yl-dithiobenzoate $(0.083 \mathrm{~g}, 0.374 \mathrm{mmol})$ and the polymerization initiator AIBN $(0.0123 \mathrm{~g}, 0.0748 \mathrm{mmol})$ were dissolved in anhydrous toluene $(5 \mathrm{~mL})$ in a glass tube, The degree of polymerization was controlled by a molar ratio of monomers and chain transfer agent.

Three freeze-pump-thaw cycles were applied to remove oxygen and humidity from solvent before the reaction. During the reaction, the glass tube was bubbled with nitrogen and immersed into thermostatic oil bath thermo-stabilized at $70^{\circ} \mathrm{C} .{ }^{1}$ After $24 \mathrm{~h}$ polymerization, the tube was inserted into liquid nitrogen to quench the reaction. The product was poured into a large excess of methanol $(\sim 500 \mathrm{~mL})$ (analytical purity, Beijing Chemical Reagents Co.) to precipitate poly (hexyl methacrylate-random-2,3dioxaborolanepropyl methacrylate). After centrifuging, the precipitant was dissolved in $\mathrm{CHCl}_{3}$ (analytical purity, Beijing Chemical Reagents Co.) and reprecipitated in excess of methanol for purification. The final product was dried in vacuum at $80{ }^{\circ} \mathrm{C}$ for more than one week before measurements or reaction. The results of the ${ }^{1} \mathrm{H}$ NMR analysis of the representative precursor 1.9-0 are shown in Figure S3. 


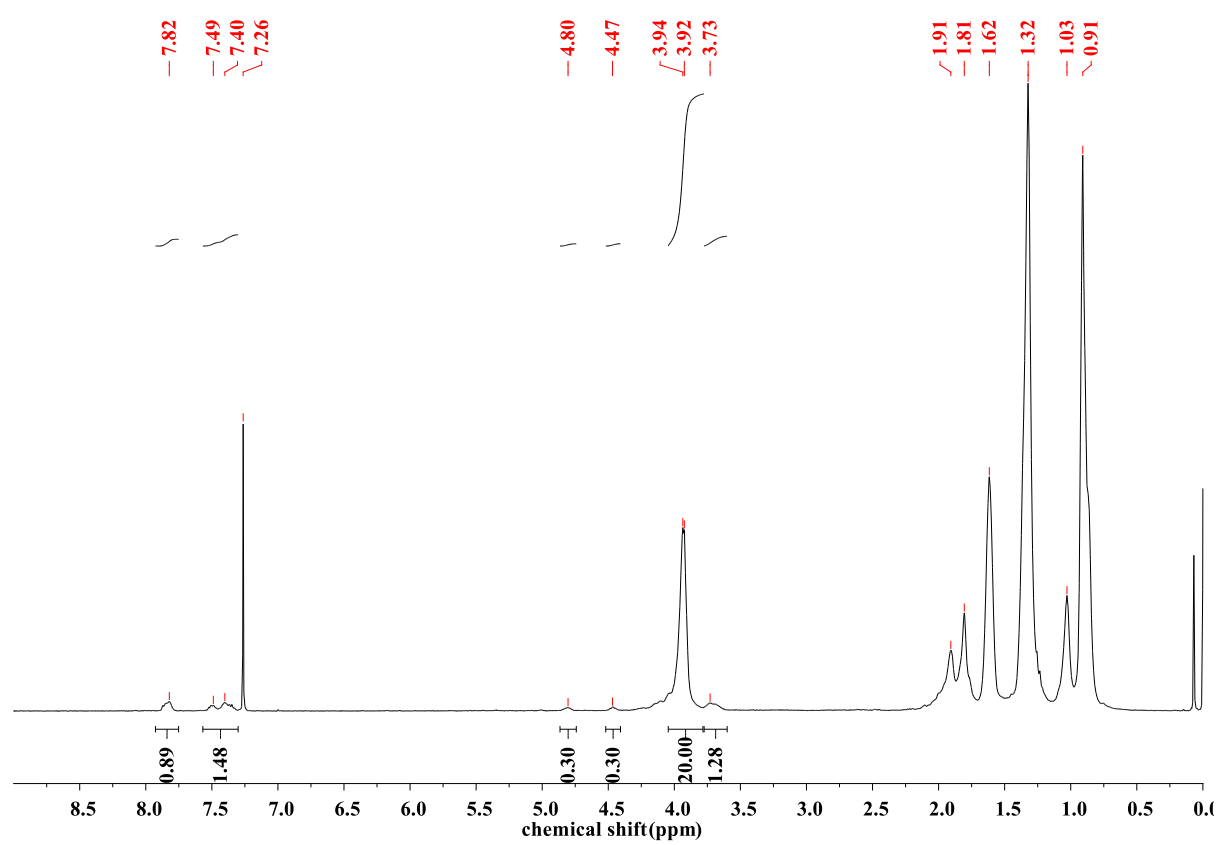

Figure S3. ${ }^{1} \mathrm{H}$ NMR spectra of poly (hexyl methacrylate-random-2,3dioxaborolanepropyl methacrylate)

2.3 Synthesis of crosslinker 2,2'-(1,4-Phenylene)-bis[4-methyl-1,3,2-dioxaborolane]

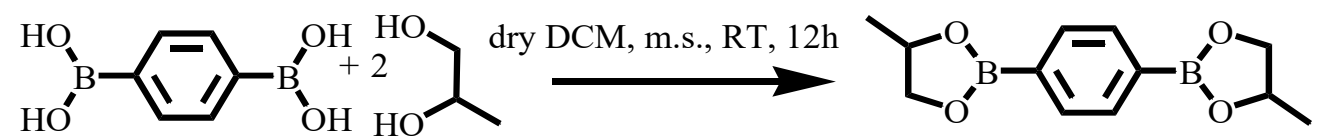

Scheme S3. The synthesis route of crosslinker

Benzene-1,4-diboronic acid (2g, 12mmol) and 1,2-propanediol $(0.83 \mathrm{~g}, 10.91 \mathrm{mmol})$ were stirred in $50 \mathrm{~mL}$ dry DCM with $10 \mathrm{~g}$ molecular sieves in a flask at room temperature. After reaction for $12 \mathrm{~h}$, the crude product was purified by filtering, centrifuging, filtering, and condensation into the final product, a bulk of white solid. The product was characterized by ${ }^{1} \mathrm{H}$ NMR and ${ }^{13} \mathrm{C}$ NMR spectroscopy as shown in Figures S4 and S5, respectively. ${ }^{1} \mathrm{H}$ NMR (400 MHz, $\left.\mathrm{CDCl}_{3}\right)$ : $\delta(\mathrm{ppm}) 7.82(\mathrm{~s}, 4 \mathrm{H})$, 4.69-4.77 (m, 2H), 4.44-4.48 (t, 2H), 3.88-3.92 (m, 2H), 1.41-1.43(d, 6H). ${ }^{13} \mathrm{C}$ NMR $\left(100 \mathrm{MHz}, \mathrm{CDCl}_{3}\right): \delta(\mathrm{ppm}) 133.8,133.8,133.8,133.8,73.6,73.6,72.4,72.4,21.5$, 21.5. The boron-bound carbon was not detected due to quadrupolar relaxation. 


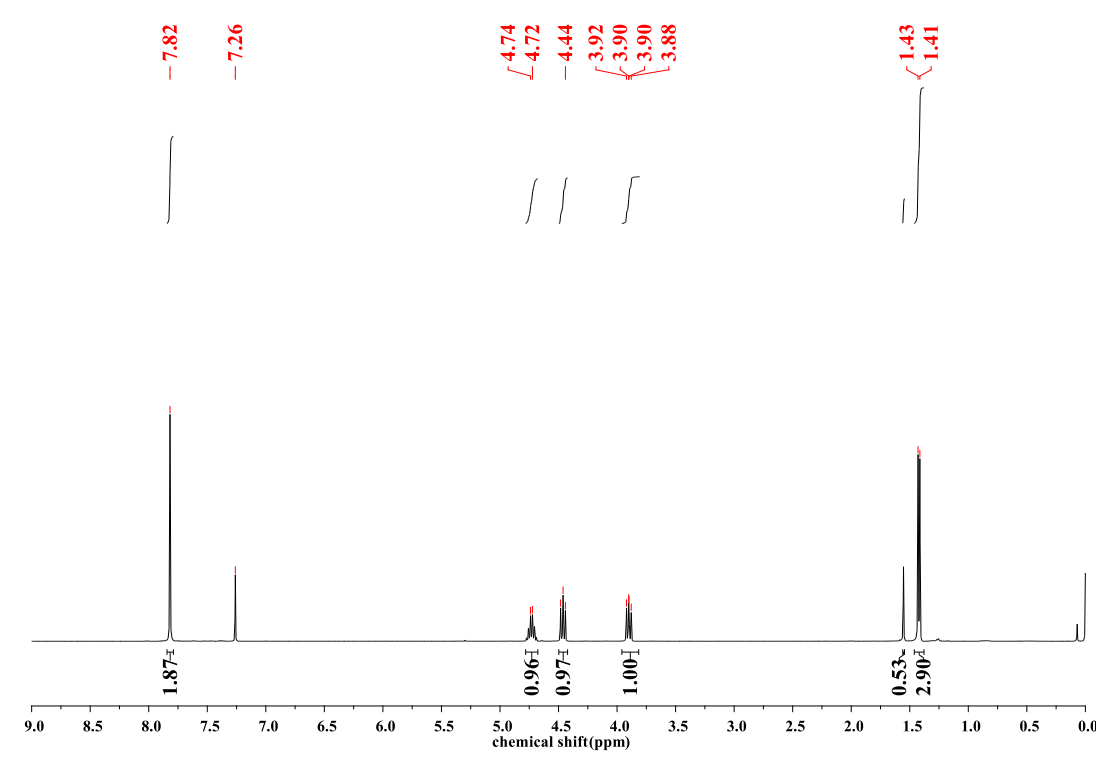

Figure S4. ${ }^{1} \mathrm{H}$ NMR spectra of crosslinker 2,2'-(1,4-Phenylene)-bis[4-methyl-1,3,2dioxaborolane]

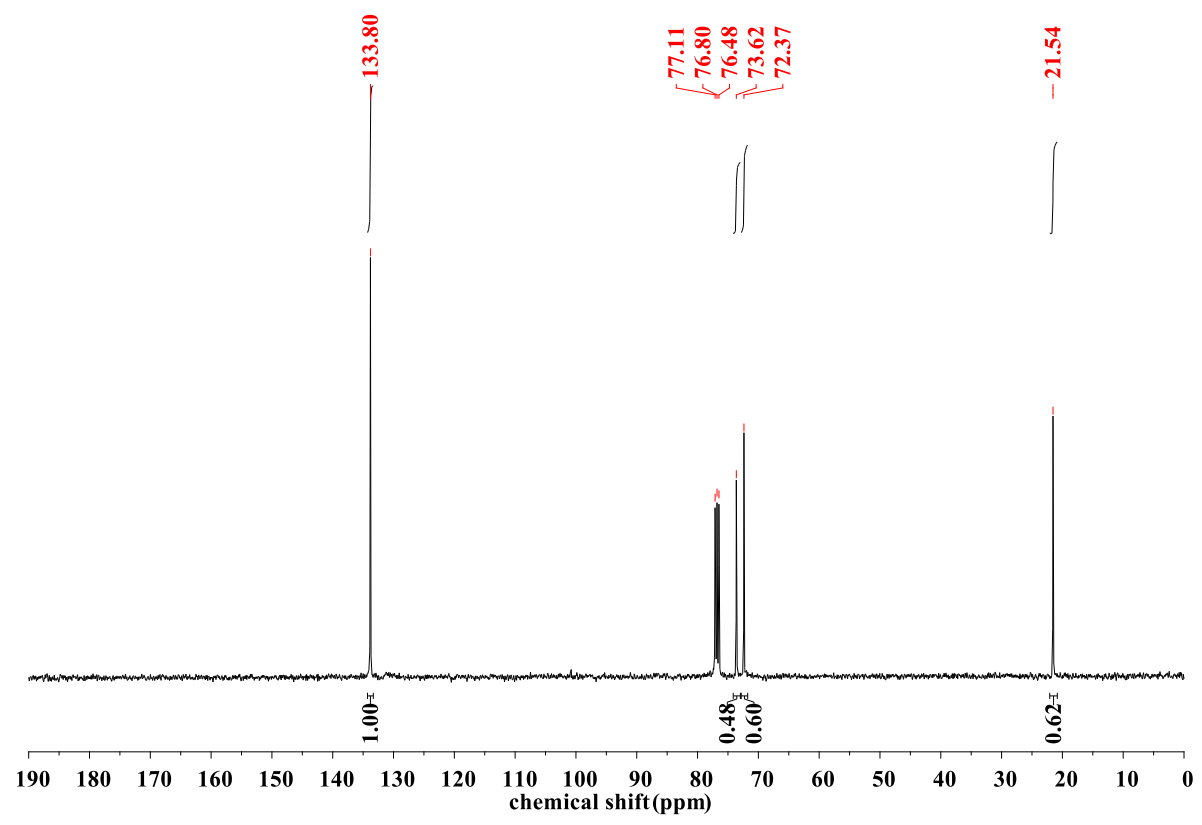

Figure S5. ${ }^{13} \mathrm{C}$ NMR spectra of crosslinker 2,2'-(1,4-Phenylene)-bis[4-methyl-1,3,2dioxaborolane]

2.4 Synthesis of vitrimer samples

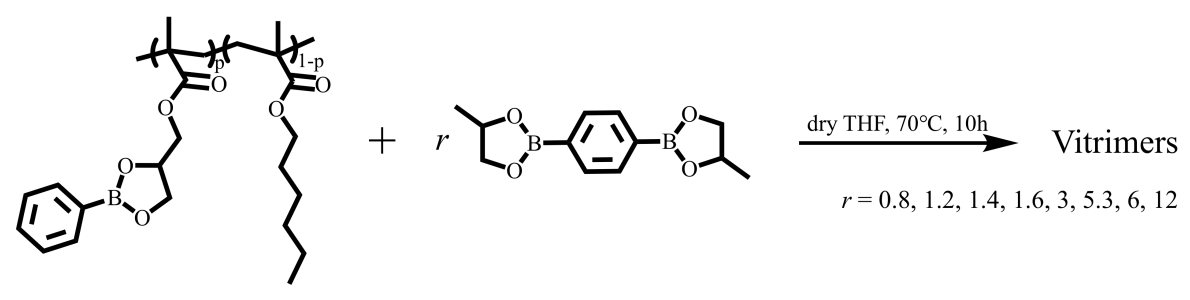

Scheme S4. The synthesis route of vitrimer samples 
As an example, we show the preparation procedure of 1.9- $r$ vitrimer samples. Poly (hexyl methacrylate-random-2,3-dioxaborolanepropyl methacrylate) $(0.3 \mathrm{~g}, 0.025 \mathrm{mmol})$ was separated into different portions, which were mixed with 2,2'-(1,4-Phenylene)bis[4-methyl-1,3,2-dioxaborolane] in anhydrous $\operatorname{THF}(10 \mathrm{~mL})$ at molar ratios of 1:0.8, $1: 1.2,1: 1.4,1: 1.6,1: 3.0,1: 5.3,1: 6.0,1: 12$, respectively, and stirred in $10 \mathrm{~mL}$ anhydrous THF in a flask at $60^{\circ} \mathrm{C}$. After $10 \mathrm{~h}$, the dioxaborolane metathesis reaction was completed. The products were dried in oven at $80^{\circ} \mathrm{C}$ for $\sim$ one week.

\section{Sample Characterization}

${ }^{1} \mathrm{H}-\mathrm{NMR}$ and ${ }^{13} \mathrm{C}-\mathrm{NMR}$ spectra were recorded with a Brucker AV $400-\mathrm{MHz}$ spectrometer using $\mathrm{CDCl}_{3}$ as a solvent and trimethylsilane as an internal standard.

Boron elemental analysis was measured with inductively coupled plasma optical emission spectroscopy (ICP-OES) analysis conducted with a Thermo iCAP spectrometer.

Gel permeation chromatography (PL-GPC) measurements were performed at ambient temperature on Wyatt Optilab equipped with low-angle light scattering and reflective index monitors to determine the molecular weight and polydispersity index $\left(M_{\mathrm{w}} / M_{\mathrm{n}}\right)$ of the precursors, poly (hexyl methacrylate-random-2,3-dioxaborolanepropyl methacrylate), as shown in Table 1 of the main text. Combination of boron elemental analysis and GPC data enabled us to determine the numbers of functional sites per chain to be $0.7,1.9$, and 3.1 for the three precursors.

Differential scanning calorimetry (DSC) analysis was performed with a DSC Q20 calorimeter (TA, USA). The samples were examined in three heating runs in the temperature range of -80 to $200^{\circ} \mathrm{C}$ at heating and cooling rates of $10^{\circ} \mathrm{C} / \mathrm{min}$. The glass transition temperature was determined from the third heating run by utilizing the commercially available Universal Analysis Software.

X-ray Scattering profiles were collected using the Rigaku SmartLab X-ray diffractometer. The diffractometer was equipped with a copper target $(2.2 \mathrm{~kW})$, a CBO mirror (Kal line, $\lambda=0.15406 \mathrm{~nm}$ ), a motorized sample stage, and a multi-slit scintillation detector. X-ray scattering patterns were recorded in a scattering angle $(2 \theta)$ 
ranging from $0.5^{\circ}$ to $25^{\circ}$ at a step-scan rate of $0.05^{\circ}$ per $5 \mathrm{~s} . \theta$ is half of the scattering angle, corresponding to the scattering vector $q=(4 \pi / \lambda) \sin \theta$ of $0.35-17.67 \mathrm{~nm}^{-1}$.

\section{Linear viscoelasticity}

Figure S6 compares a change of (pseudo-) master curve of $G^{\prime}$ and $G^{\prime \prime}$ with $r$ for the 0.7 $r$ samples. The degree of gelation first increases with $r$ for $r \leq 2$, while decreases when $r$ is further increased to 6.0. Since a number of functional sites per chain $N p(=0.7)$ is very low, most of chains contain only zero or one functional site (cf. Figure 2 in the main text). No gel behavior is observed with an increase of $r$.
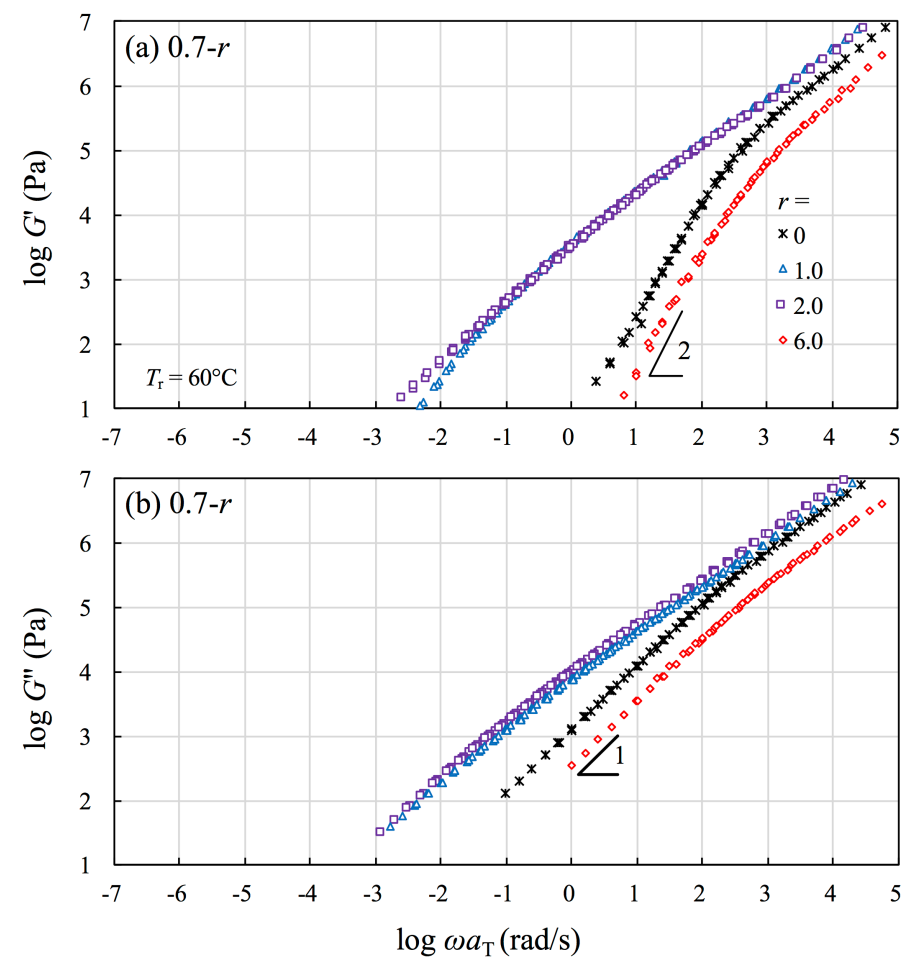

Figure S6. Comparison of pseudo-master curves of the storage and loss moduli, $G^{\prime}$ and $G^{\prime \prime}$, reduced at $T_{\mathrm{r}}=60^{\circ} \mathrm{C}$ for the vitrimer samples $0.7-r$ with $r$ from 0 to 6 .

Figure S7 compares a change of (pseudo-) master curve of $G^{\prime}$ and $G^{\prime \prime}$ with $r(\leq 2)$ for the 3.1-r samples. Since the number of functional sites per chain becomes sufficiently high, clear sol-to-gel transition due to the crosslinking is observed. Further increase of $r$ leads to the decrosslinking and accordingly a gel-to-sol transition, as seen in Figure 
S8. All these features are very similar to those in Figures 6 and 7 of the 1.9-r samples in the main text.
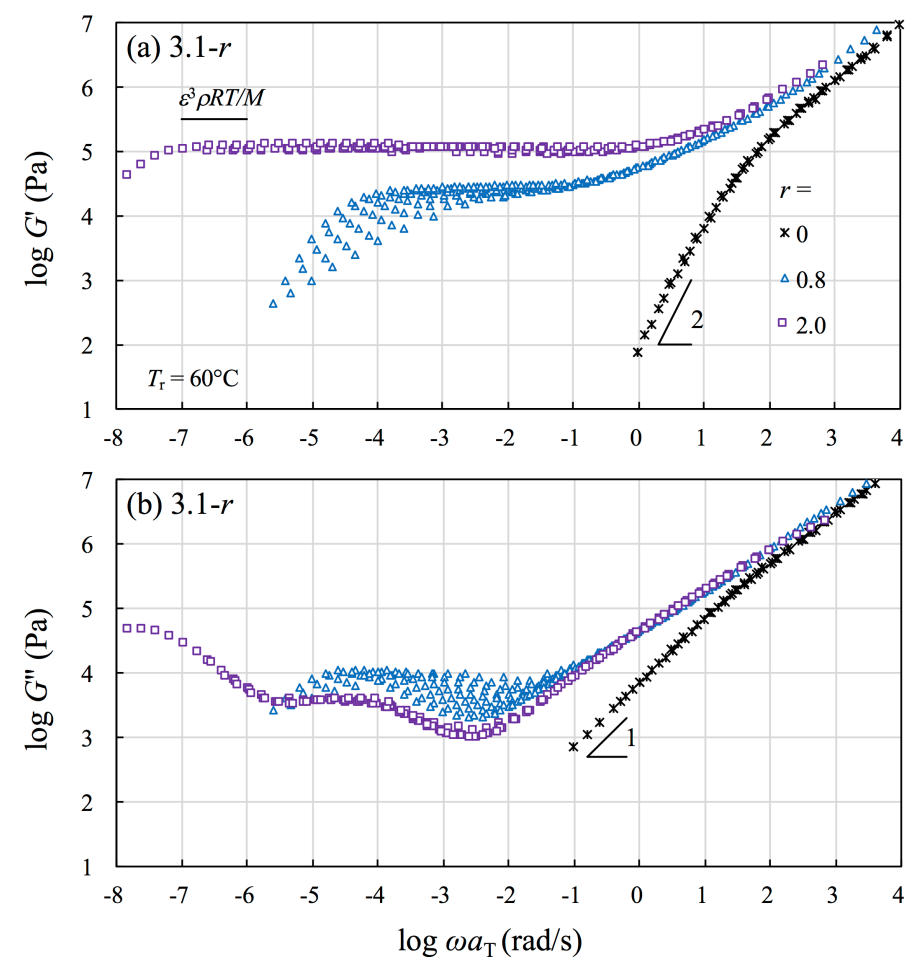

Figure S7. Comparison of pseudo-master curves of the storage and loss moduli, $G^{\prime}$ and $G^{\prime \prime}$, reduced at $T_{\mathrm{r}}=60^{\circ} \mathrm{C}$ for the vitrimer samples 3.1- $r$ with $r$ from 0 to 2.0.
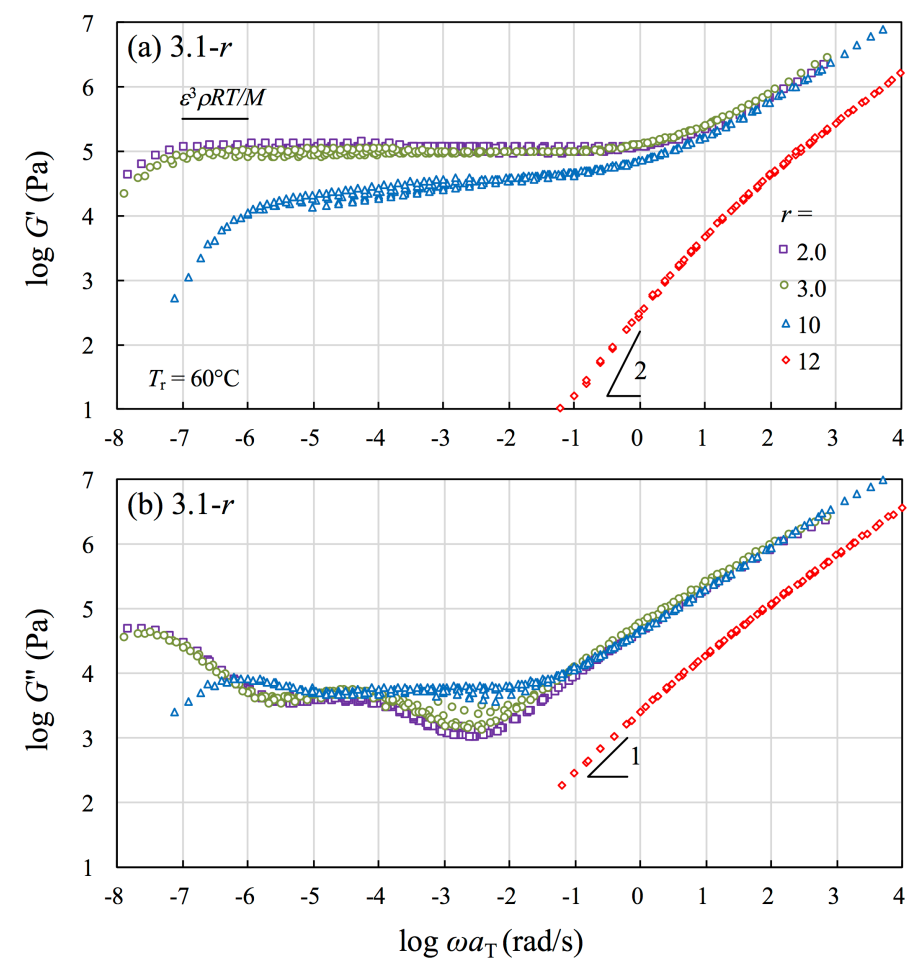

Figure S8. Comparison of pseudo-master curves of the storage and loss moduli, $G^{\prime}$ and $G^{\prime \prime}$, reduced at $T_{\mathrm{r}}=60^{\circ} \mathrm{C}$ for the vitrimer samples 3.1-r with $r$ from 2.0 to 12 . 
Figure S9 shows the storage and loss moduli, $G^{\prime}$ and $G^{\prime \prime}$, shifted by factors (a) $a_{\mathrm{T}}$ under guidance of the Rouse part of moduli and (b) $a_{\mathrm{T}}{ }^{\prime}$ under the guidance of the decrosslinking part of moduli for the 1.9-1.6 sample. The different shifting methods of panels (a) and (b) are explained in detail in the main text. The overall trend seen in Figure S9 is in spirit similar to that of the 1.9-1.4 sample shown in the main text.

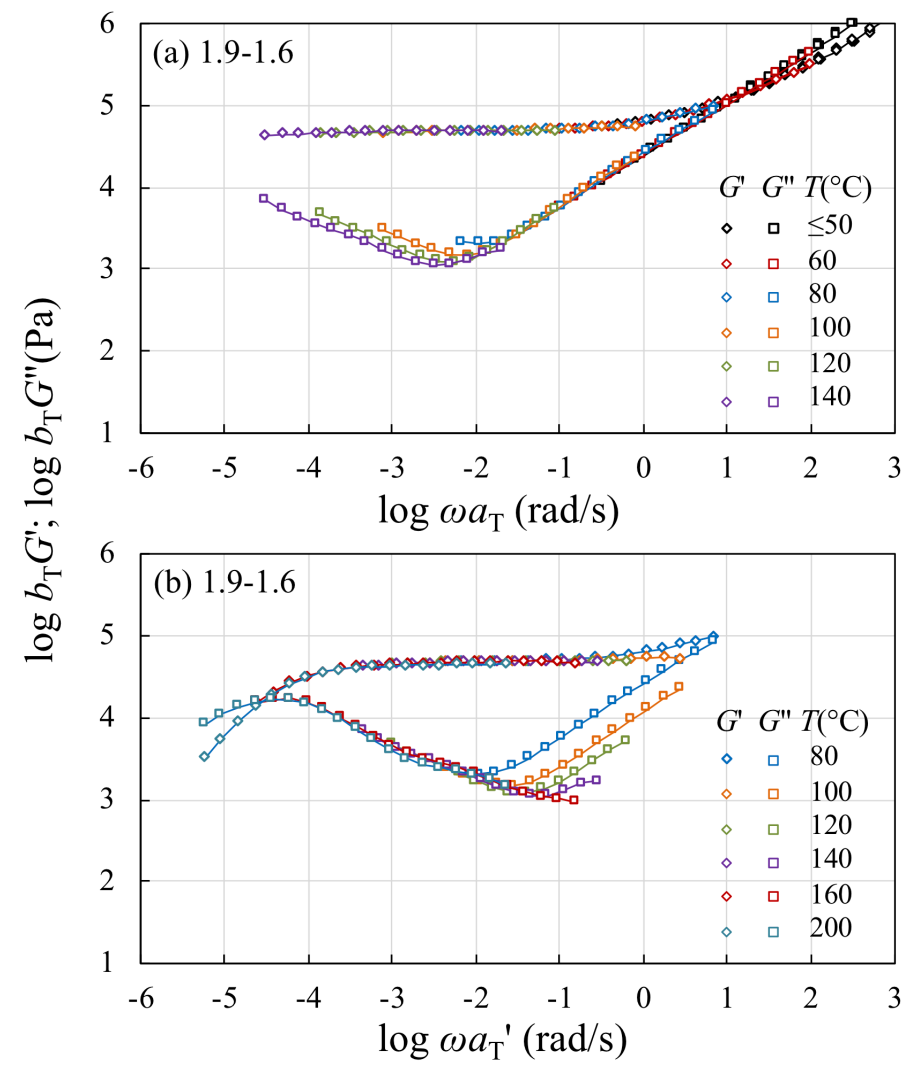

Figure S9. Test of time-temperature superposition of the 1.9-1.6 sample. The storage and loss moduli $G^{\prime}$ and $G^{\prime \prime}$ are normalized by a temperature factor $b_{\mathrm{T}}=T_{\mathrm{r}} / T$, and shifted by (a) $a_{\mathrm{T}}$ to superpose the high- $\omega$ part of modulus arising from the Rouse part of modulus, and (b) $a_{\mathrm{T}}$ to superpose the low- $\omega$ part of modulus arising from the decrosslinking to that at $T=80^{\circ} \mathrm{C}$ where $a_{\mathrm{T}}{ }^{\prime}=a_{\mathrm{T}}$.

\section{X-ray Scattering}

Figure S10 shows X-ray scattering profiles of representative samples, where two correlation peaks are commonly seen for all samples, the high $q$ amorphous peak and the low $q$ backbone-backbone correlation peak. The similarity of these profiles suggests that the crosslinker molecules are well dispersed in the vitrimer samples. 


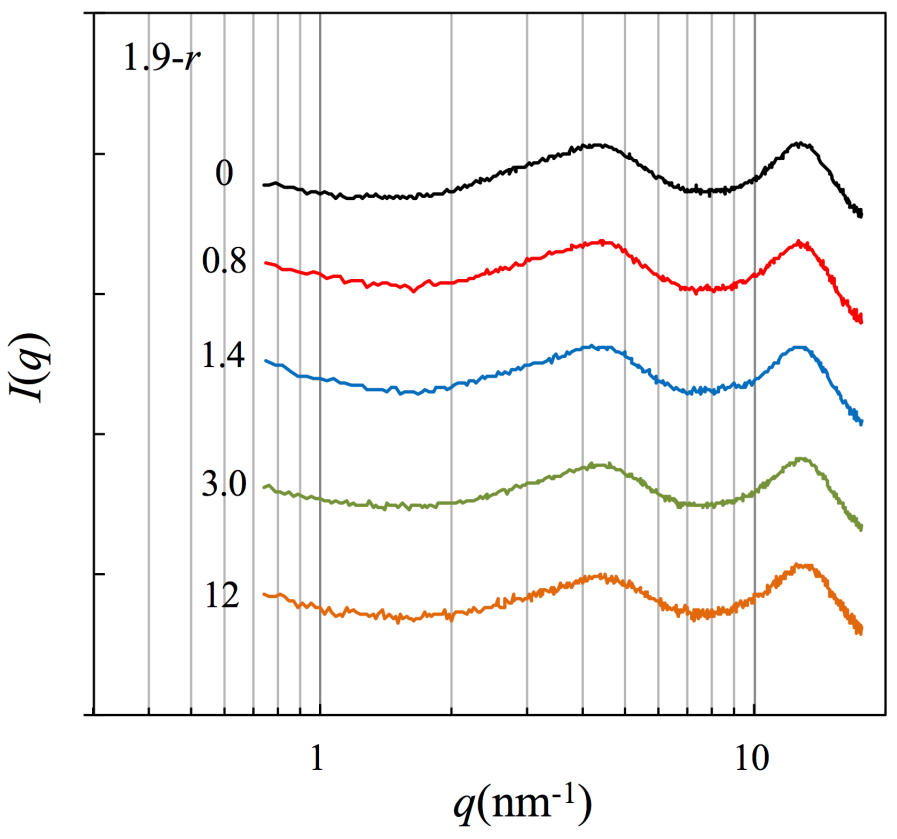

Figure S10. Comparison of X-ray scattering profiles of the 1.9-r samples with $r=0$, $0.8,1.4,3.0$, and 12. The high and low $q$ peaks are attributed to the amorphous scattering of the side chains and the backbone correlation, respectively.

\section{Reference}

1. Cash, J. J.; Kubo, T.; Bapat, A. P.; Sumerlin, B. S. Room-Temperature Self-Healing Polymers Based on Dynamic-Covalent Boronic Esters. Macromolecules 2015, 48, (7), 2098-2106. 